\title{
Geochemical databases and a need for linkages to other database systems
}

\author{
B.M. EGLINGTON ${ }^{1}$, S.J. PeHRSSON ${ }^{2}$ AND D.L. HUSTON ${ }^{3}$ \\ ${ }^{1}$ University of Saskatchewan, Canada. bruce.eglington@usask.ca \\ ${ }^{2}$ Geological Survey of Canada, Ottawa \\ ${ }^{3}$ Geoscience Australia, Canberra
}

Geochemical and other geoscience data systems, whether structured as databases or less formally, are essential to the efficient study of Earth systems. Numerous database systems exist for geochemistry, a few for palaeontology and geochronology and several geological surveys run lithostratigraphic databases and provide GIS maps. Very few of these are, however, designed or implemented connect across discipline-specific divides. It is essential that future efforts be directed towards facilitating interconnectivity of the various systems so as to provide mechanisms to routinely interogate data in a multidisciplinary sense. Although developed on a relatively small scale compared to available geochemical and palaeontological databases, systems designed for use in the IGCP 509 and 648 projects illustrate some of the advantages of designing interconnectivity at the root of the system. These systems provide mechanisms to link lithostratigraphy to GIS maps, to geochronology, isotope geochemistry, palaeomagnetic data, ore deposit information, geochemistry and other geolscience data and to palaeogeographic reconstructions. As developers of theses systems we are well aware that there is much to be learned from other developers and that the scale of the problem facing us all requires collaborative development according to mutually beneficial best practices. Interconnectivity, not just to be able to read data from multiple platforms, but also to query across linked discipline-specific topic, needs to become part of the design process. Quality control will never succeed with purely computer-based artificial intelligence systems. Rather, we need to leverage the best quality checkers that already exist, experts in the various sub-disciplines of the earth sciences by developing systems which they routinely use and so identify and correct occasional mistakes which get missed by the more automated systems. 